\title{
Research on Percolation Threshold of Broadband Electromagnetic Wave Absorbing SiCN (MWCNTs) Composite Ceramics
}

\section{Shan Wang}

Jilin University

Hongyu Gong

Shandong University

Desheng Qi

Jilin University

M. Zeeshan Ashfaq

Shandong University

Xigui Yue ( $\nabla$ yuexigui@jlu.edu.cn )

Jilin University

\section{Research Article}

Keywords: polymer derivation method, SiCN-based composite ceramics, percolation threshold, conductive network, electromagnetic wave absorption performance

Posted Date: October 12th, 2021

DOI: https://doi.org/10.21203/rs.3.rs-955134/v1

License: (c) (i) This work is licensed under a Creative Commons Attribution 4.0 International License. Read Full License 


\title{
Research on Percolation Threshold of Broadband Electromagnetic Wave
}

\author{
Absorbing SiCN (MWCNTs) Composite Ceramics \\ Shan Wang ${ }^{1}$, Hongyu Gong ${ }^{2,3 * *}$, Desheng $\mathrm{Qi}^{1}$, M. Zeeshan Ashfaq ${ }^{2,3}$, Xigui Yue $^{1 *}$ \\ ${ }^{1}$ Alan G. MacDiarmid Institute, College of Chemistry, Jilin University, 2699 Qianjin \\ street, Changchun 130012, People's Republic of China \\ ${ }^{2}$ Key Laboratory for Liquid-Solid Structural Evolution \& Processing of Materials of \\ Ministry of Education, School of Materials Science and Engineering, Shandong \\ University, Jingshi Road 17923, Jinan 250061, People's Republic of China \\ 3Key Laboratory of Special Functional Aggregated Materials, Ministry of Education, \\ School of Materials Science and Engineering, Shandong University, Jingshi Road \\ 17923, Jinan 250061, People’s Republic of China \\ Corresponding author*: yuexigui@jlu.edu.cn \\ Corresponding author**: gong_hongyu@163.com
}

\begin{abstract}
A simple polymer derivation method was applied in this paper to prepare a series of SiCN (MWCNTs) composite ceramics by adjusting the mass ratio of multi-walled carbon nanotubes (MWCNTs). The percolation threshold of the corresponding MWCNTs addition amount when SiCN(MWCNTs) composite ceramics exhibit the optimal electromagnetic wave (EWM) absorption performance was studied, the effect of different addition amounts of MWCNTs on reflection loss (RL), effective absorption bandwidth $(\mathrm{EAB})$, electromagnetic parameters, impedance matching parameters $(Z)$ and attenuation coefficient $(\alpha)$ of composite ceramics was analyzed,
\end{abstract}


and the EMW absorption mechanism of the corresponding composite ceramics when MWCNTs addition amount fix at percolation threshold was discussed. The results showed that composite ceramics exhibited the best EMW absorption performance when the addition amount of MWCNTs reached the percolation threshold (10wt $\%)$ : the minimum reflection loss $\left(\mathrm{RL}_{\mathrm{min}}\right)$ was $-37.9 \mathrm{~dB}$, and the EAB was $2.8 \mathrm{GHz}$ at a thickness of $2.4 \mathrm{~mm}$; its $\mathrm{RL}_{\min }$ was $-21.7 \mathrm{~dB}$, and the $\mathrm{EAB}$ reached $4.7 \mathrm{GHz}$ at a thickness of $1.7 \mathrm{~mm}$. By changing the sample thickness from $1.0 \mathrm{~mm}$ to $5.0 \mathrm{~mm}$, the EAB containing the $\mathrm{C}, \mathrm{X}$ and $\mathrm{Ku}$ bands can be acquired. Therefore, it is expected to be a promising candidate for the new generation of EMW absorbers due to its light weight, high efficiency and broad band.

Keywords: polymer derivation method; SiCN-based composite ceramics; percolation threshold; conductive network; electromagnetic wave absorption performance

\section{Introduction}

The continuous development of modern technology and the advent of the 5G era lead to that electromagnetic wave (EMW) pollution has quickly evolved into an environmental issue closely concerned by the world [1]. Nowadays, various countries have increased the research on EMW pollution, and related standards are being developed to purify the space contaminated by EMW. In order to prevent EMW from harming human body and polluting environment, simple and effective measures commonly used are to absorb EMW by EMW absorbers and deplete electromagnetic energy in the material, which can reduce redundant EMW in the space to achieve the 
purpose of purifying the environment and ensuring human health [2]. In recent years, considerable attention has been paid to SiCN ceramics in the field of EMW absorbers [3]. Relevant studies have shown that $\mathrm{SiCN}$ ceramics can withstand a high temperature of $1100{ }^{\circ} \mathrm{C}$ in air atmosphere without changes in morphology and structure, and this excellent high temperature resistance and oxidation resistance is absent in polyester-based and carbon-based EMW absorbers [4]. Beyond that, the carbon nanodomain contained in it ensures a good dielectric loss performance and a certain EMW absorption capacity (SiCN ceramics with thickness of $8 \mathrm{~mm}$ can absorb about $90 \%$ EMW in the range of $6 \sim 16 \mathrm{GHz}$. It is also a good EMW absorber while serving as a dispersible particle absorber for matrix), which is rare in most high-temperature resistant ceramics, so $\mathrm{SiCN}$ ceramics are considered as potential candidates in the field of EMW absorbers [3]. However, compared with polyester-based and carbon-based EMW absorbers, SiCN ceramics have the disadvantage of narrow effective absorption bandwidth (EAB) at a small thickness, which greatly limits its use in harsh environment $[2,3,5,6]$. To this end, reducing the thickness of SiCN ceramics and increasing its $\mathrm{EAB}$ under the condition of ensuring the EMW absorption rate is of great research significance and practical application value.

Carbon nanotubes (CNTs) have a similar structure to graphite, and exhibit a good electrical conductivity, which is attributed to that $\pi$ electrons contained in CNTs can move freely in the non-localized conjugated system formed within and on its surface [7,8]. The introduction of CNTs into the ceramic matrix as inclusions can improve the 
conduction loss and EMW absorption capacity of composite ceramics, which has been demonstrated in our previous work [9]. However, too few addition amount of inclusions (conductive materials) is not conductive to the formation of a three-dimensional conductive network, which will lead to electron transport lag and thus affect the EMW absorption performance; ceramics may become highly conductive materials because of excessive addition amount, which will result in too large permittivity of composite ceramics and impedance mismatch, and thus deteriorate the EMW absorption performance [2,9]. When the concentration of inclusions in the composite materials reaches a certain value, the phenomenon of sudden and substantial improvement of performance parameters of the composite material is referred to as percolation phenomenon, and the concentration value of the corresponding inclusions is called "percolation threshold"; When the content of inclusions exceeds the percolation threshold, the performance of the composite material will not improve or will deteriorate [10-12]. In the composite ceramic system, if the EMW absorption performance of composite ceramics can be greatly improved by using the minimum conductive filler (namely, the percolation threshold), it will have a great stimulative effect on reducing production cost and providing a better environment for the society.

Accordingly, in order to explore the minimum filling rate of conductive filler (namely, the percolation threshold of conductive filler) when composite ceramics exhibit the optimal EMW absorption performance, Multi walled carbon nanotubes (MWCNTs) with good electrical conductivity were introduced into SiCN ceramics by 
the polymer derivation in this study. A conductive percolation network was gradually formed in the SiCN ceramic matrix by adjusting the addition amount of MWCNTs (3wt\% 30wt\%). On this basis, the percolation threshold of MWCNTs for composite ceramics with the best EMW absorption performance was analyzed, and the effect of the change in the addition amount of MWCNTs on the EMW absorption performance, permittivity, impedance matching parameters $(\mathrm{Z})$ and attenuation constant $(\alpha)$ of $\operatorname{SiCN}$ (MWCNTs) composite ceramics was discussed. In this paper, the formation conditions of conductive percolation state inside SiCN ceramics and the EMW absorption performance and mechanism of composite ceramics under such conditions were analyzed for the first time, which provides a new idea for optimizing the EMW absorption performance of SiCN-based composite ceramics.

\section{Experiment}

A simple polymer derivation was applied in this experiment. First, liquid polysilazane (PSZ, HT-1800) was cross-linked for $2 \mathrm{~h}$ in a tubular furnace with nitrogen at $600{ }^{\circ} \mathrm{C}$ to obtain the cross-linked precursor, which was then ball-milled to get the precursor powder. Next, MWCNTs (ID: 5 10 nm, OD: $10 \sim 30 \mathrm{~nm}$, purity: $>90 \%)$ of different mass ratios $(0 \mathrm{wt} \%, 3 \mathrm{wt} \%, 5 \mathrm{wt} \%, 7 \mathrm{wt} \%, 9 \mathrm{wt} \%, 10 \mathrm{wt} \%$, $15 \mathrm{wt} \%, 20 \mathrm{wt} \%, 25 \mathrm{wt} \%, 30 \mathrm{wt} \%)$ were mixed with precursor powder by ball milling and then pressed into disc-shaped tablets. Finally, the tablets were pyrolyzed for $2 \mathrm{~h}$ in a tubular furnace with nitrogen at $1100{ }^{\circ} \mathrm{C}$ to obtain $\mathrm{SiCN}$ (MWCNTs) composite ceramics (abbreviated as SiCN (MWCNTs)). In the following, SiCN (MWCNTs) with $3 \mathrm{wt} \%, 5 \mathrm{wt} \%, 7 \mathrm{wt} \%, 9 \mathrm{wt} \%, 10 \mathrm{wt} \%, 15 \mathrm{wt} \%, 20 \mathrm{wt} \%, 25 \mathrm{wt} \%, 30 \mathrm{wt} \%$ addition amount 
of MWCNTs were respectively named samples 3, 5, 7, 9, 10, 15, 20, 25, 30, and the SiCN ceramic (abbreviated as $\mathrm{SiCN}$ ) with $0 \mathrm{wt} \%$ addition amount of MWCNTs was named sample 0 to facilitate the analysis. The experimental flow chart is shown in Fig.

1. The test equipment information of the sample is shown in Table 1.

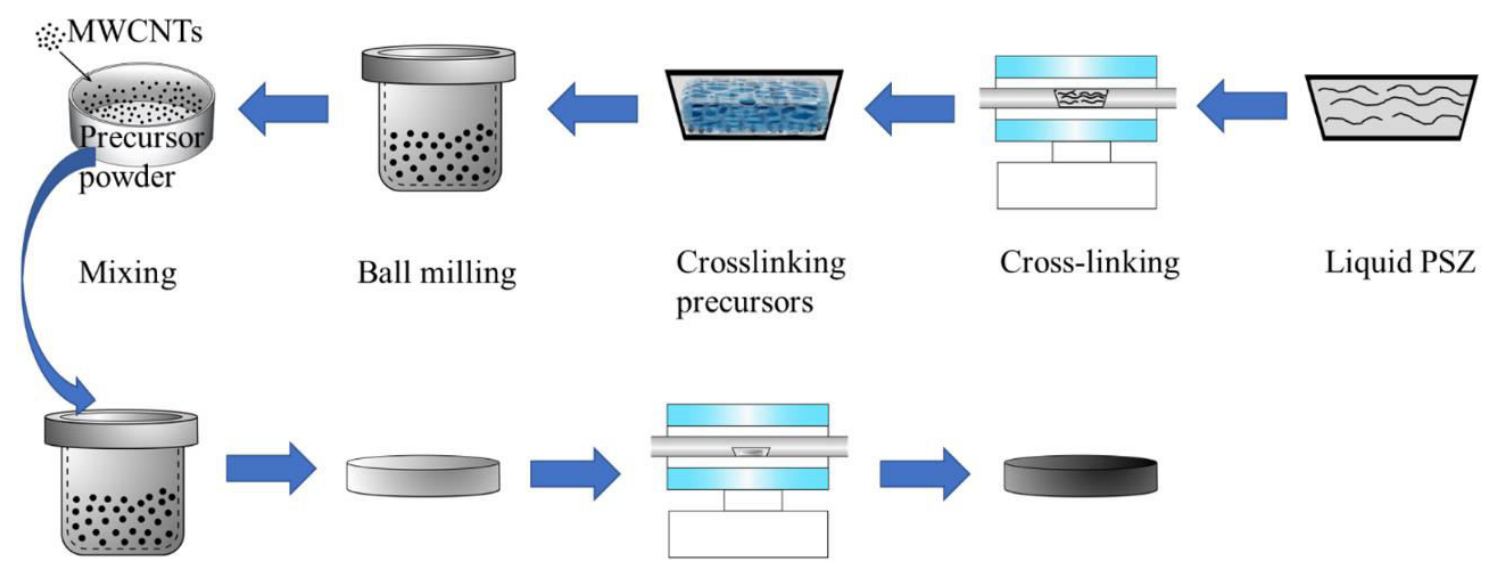

Fig.1 Preparation Process Diagram of SiCN (MWCNTs)

\begin{tabular}{lc}
\hline Equipment & Equipment information \\
\hline XRD & D/MAX-Ultima IV, Rigaku, Japan \\
Raman spectrometer & Renishaw, UK \\
SEM & EVO18, Zeiss, German \\
TEM & JEM-1200EX, JEOL, Japan \\
XPS & PHI1600EXCA, UK \\
Vector network analyzer & E5071C, Keysight, USA \\
\hline
\end{tabular}

Table 1 Test equipment list

\section{Results and Discussion}

\subsection{Phase Composition Analysis}

Fig. 2a shows the XRD pattern of each sample. The XRD image of each ceramic presented typical diffuse scattering peaks, indicating the amorphous state of ceramic samples. Compared with SiCN, SiCN (MWCNTs) showed an additional carbon (C PDF Card No 25-0806) diffraction peak near $26^{\circ}$, which is caused by the introduction 
of MWCNTs [9]. With the rise of MWCNTs content, the diffraction peak of carbon increased, indicating its elevated crystallinity. Except for the diffraction peaks of carbon, no other diffraction peaks appeared in SiCN (MWCNTs), showing that no additional phases were generated in composite ceramics. Fig. 2b is the Raman spectrum of each sample. The clear D peak and G peak in each sample indicated the existence of free carbon in ceramics, which provided media for electron transport and transition [4]. The defect level of carbon-containing materials can be characterized by the intensity ratio of $\mathrm{D}$ peak to $\mathrm{G}$ peak $\left(\mathrm{I}_{\mathrm{D}} / \mathrm{I}_{\mathrm{G}}\right)$, and a larger ratio of $\mathrm{I}_{\mathrm{D}} / \mathrm{I}_{\mathrm{G}}$ demonstrated a higher defect level in the material [2]. It was observed from Fig. 2b that the $\mathrm{I}_{\mathrm{D}} / \mathrm{I}_{\mathrm{G}}$ of SiCN (MWCNTs) was significantly increased compared with that of $\mathrm{SiCN}$, suggesting that the introduction of MWCNTs brought a great quantity of defects to SiCN (MWCNTs). In the alternating electromagnetic filed, defects can promote polarization loss as polarization centers and thus positively contribute to the attenuation of EMW [2,13].
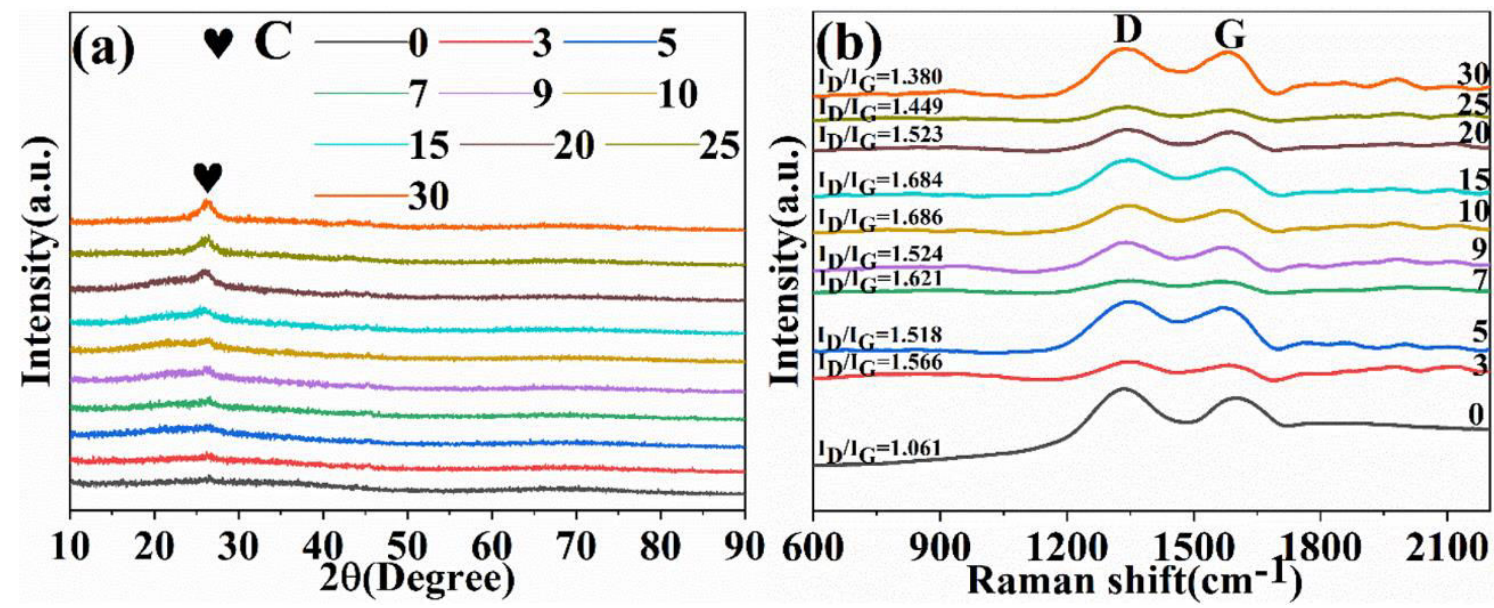

Fig. 2 XRD Pattern (a) and Raman Spectrum (b) of SiCN (MWCNTs) 

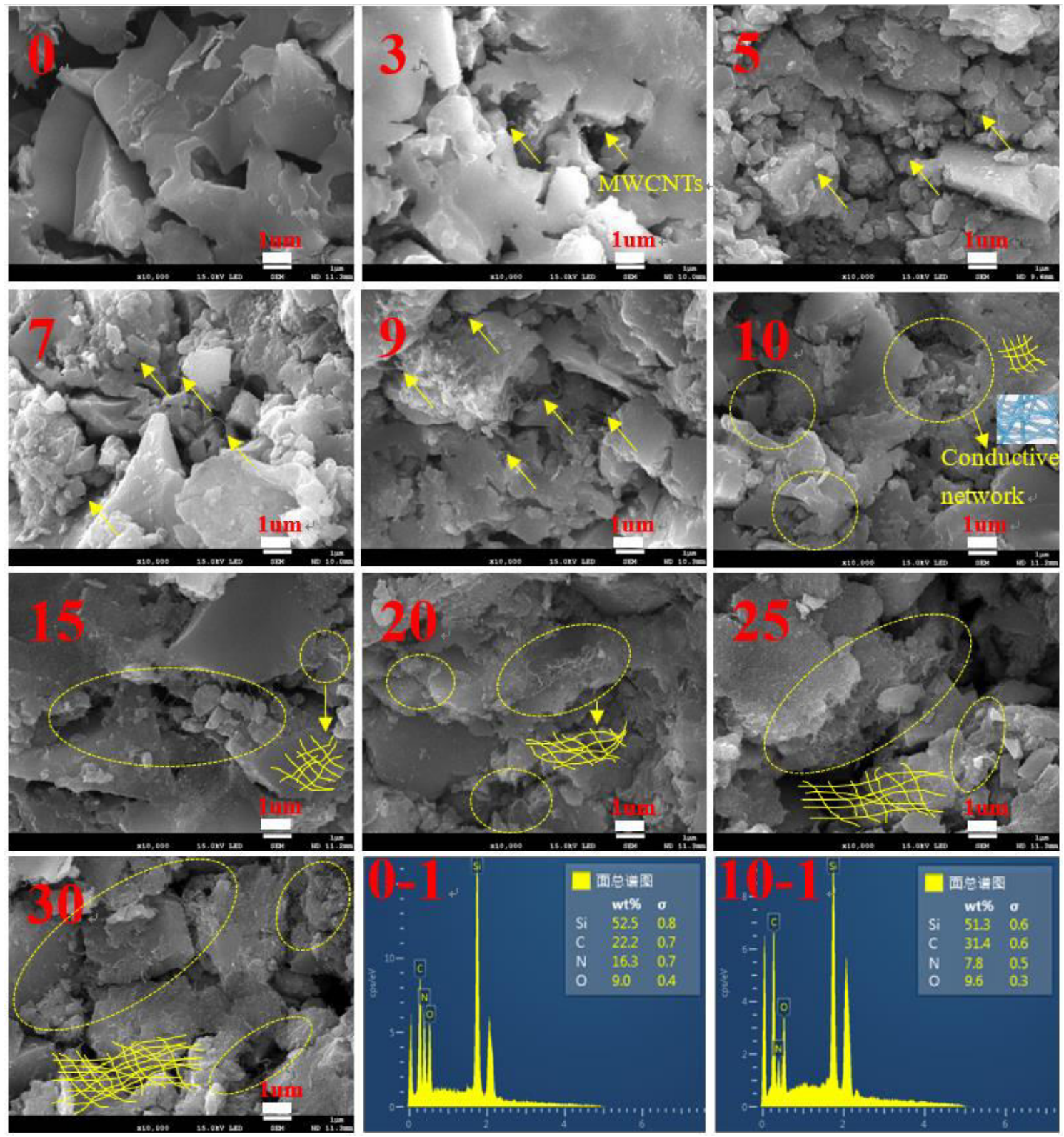

Fig. 3 SEM Images (0 30) of SiCN (MWCNTs), EDS Analysis Diagram (0-1) of

Sample 0 and EDS Analysis Diagram (10-1) of Sample 10

\subsection{Microstructure Analysis}

Fig. 3 shows the SEM images of each sample at a magnification of 10000 (Fig. 3 0 30) and the EDS analysis diagrams of sample 0 and sample 10 (Fig. 3 0-1 and Fig.

3 10-1). It can be observed from Images $0 \sim 30$ that ceramic matrices presented amorphous block-like continuous structures with a small number of holes in it. With 
the increase of MWCNT addition amount, Images 0 30 showed the evolution of conductive network formation in the ceramic matrix: At a lower content of MWCNTs, MWCNTs were not connected to each other but existed as independent conductor units; With the increase of MWCNTs content, the number of independent conductor units increased (Fig. 3 0 9). When the addition amount of MWCNTs reached 10wt\%, it was observed that MWCNTs lapped with each other (Fig. 3 10), indicating that independent conductor units were interconnected to form conductive network, which may lead to a sudden improvement in the dielectric loss performance [14]; As the addition amount of MWCNTs continued to increase, the conductive network tended to be saturated, and the phenomena that the conductor units agglomerated, and the conductive networks interlaced and covered with each other appeared(Fig. 3 15 30).

Fig. 4 schematically shows the evolution of MWCNTs in the ceramic matrix from independent random dispersion to interweaving into network to agglomeration and stacking.

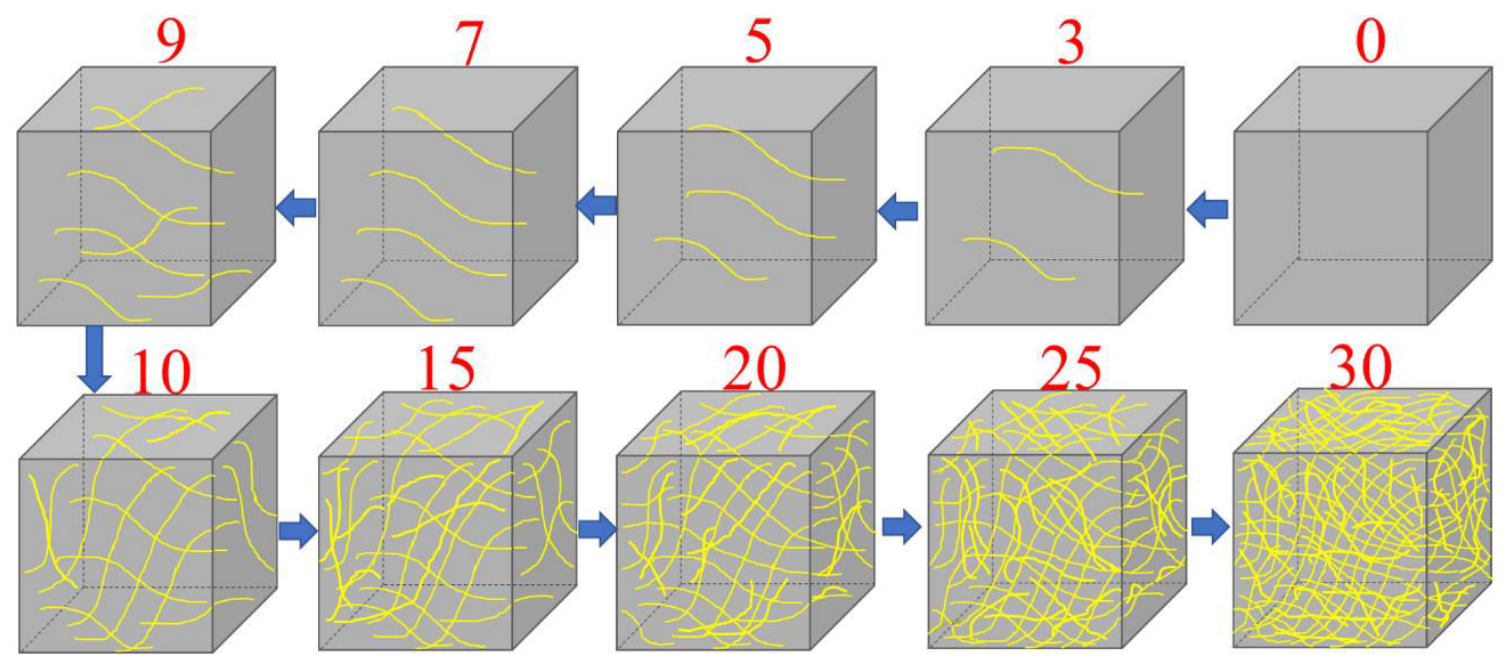

Fig. 4 Evolution Diagram of Conductive Network in SiCN (MWCNTs)

Fig. 3 0-1 shows that the elemental composition ( $\mathrm{Si}, \mathrm{C}, \mathrm{N}$ and $\mathrm{O})$ of the ceramic 
matrix. Oxygen may be introduced through the preparation process due to the absence of oxygen in PSZ [15]. Compared with sample 0, the content of C element in sample 10 increased significantly (Fig. $3 \mathbf{1 0 - 1}$ ), which is attributed to the addition of MWCNTs; while the content of $\mathrm{N}$ element decreased, which may be induced by the fact that in the composite system of SiCN (MWCNTs), Si is more easily to bond with $\mathrm{C}$ rather than $\mathrm{N}$, and the decomposition of non-bonded $\mathrm{N}$-containing groups in the pyrolysis process leads to the decrease in the content of $\mathrm{N}$ element [16]. In order to further observe the internal structure of SiCN (MWCNTs), TEM analysis was performed on sample 10, and the results are shown in Fig. 5. From the figure a multi-walled tube with a diameter of about $20 \mathrm{~nm}$ was observed to be embedded in the ceramic matrix (Fig. 5 10-1 and Fig. 5 10-2). HRTEM analysis of the tube shows that its lattice fringe distance was $0.33 \mathrm{~nm}$, which was indexed to the (004) crystal plane of C (Fig. 5 10-3), demonstrating that MWCNTs are still intact in the ceramic matrix after pyrolysis at $1100{ }^{\circ} \mathrm{C}$. A slight decrease in the diameter of MWCNT was found at the fracture (decrease from $15 \mathrm{~nm}$ to $13 \mathrm{~nm}$, Fig. 5 10-4 and Fig. 5 10-5), which suggests that there is a good interface bonding between MWCNTs and SiCN ceramic matrix, and that MWCNTs may have the effect of toughening the ceramic matrix through pull out, bridging and other mechanisms [17]. In addition to MWCNTs, circular eddy carbon can be observed in the ceramic matrix (Fig. 5 10-5). The existence of eddy carbon provides a channel for electron transport and transition, and is also the basis for $\mathrm{SiCN}$ ceramic matrix to have certain dielectric loss properties [3, 4, 18]. Fig. 5 10-6 is the selected area electron diffraction pattern of the sample. In the 
figure, the widened diffraction ring was indexed to the (004) crystal plane of C [19], and no other obvious diffraction ring was observed, indicating that no additional crystal phase exists except $\mathrm{C}$ in the amorphous ceramic matrix, which is consistent with the XRD analysis results.
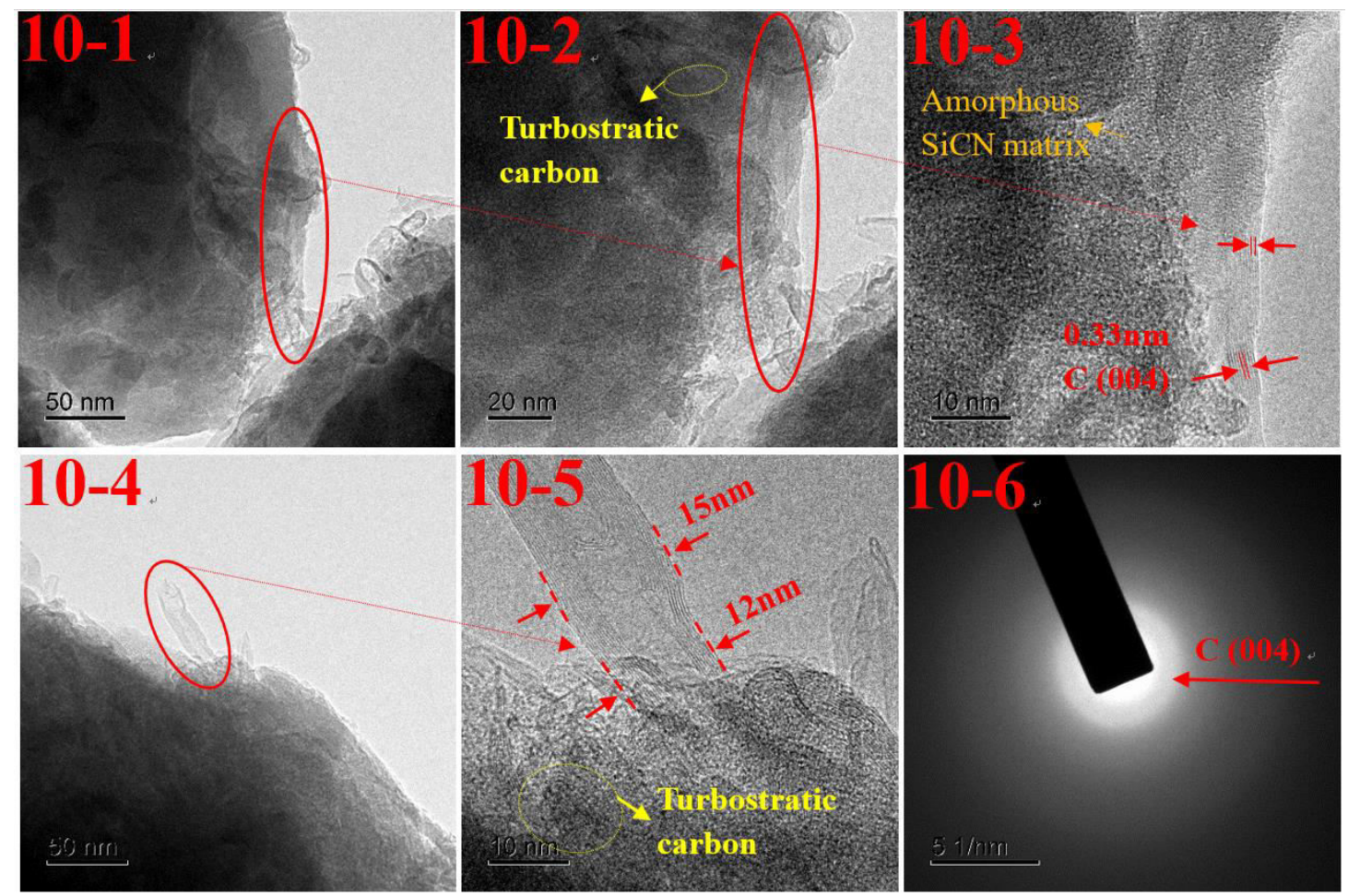

Fig. 5 TEM Images (10-1 10-5) of SiCN (MWCNTs) and Selected Area Electron Diffraction Pattern (10-6)

\subsection{Chemical Composition Analysis}

XPS can be used to analyze the chemical composition of the sample and the bonding mode of internal atoms. Fig. 6 0-1 and Fig. 6 10-1 show that elements contained in sample 0 and sample 10 were $\mathrm{Si}, \mathrm{C}, \mathrm{N}$, and $\mathrm{O}$. The Si2p peaks of sample 0 and sample 10 can be deconvoluted into $\mathrm{Si}-\mathrm{C}, \mathrm{Si}-\mathrm{N}$ and $\mathrm{Si}-\mathrm{O}$ peaks (Fig. 6 0-2 and Fig. 6 10-2). Compared with sample 0 , the amount of Si-N peaks in sample 10 decreased significantly (Fig. 6 0-2 and Fig. 6 10-2), suggesting that the introduction 
of MWCNTs changed the bonding mode of atoms in the SiCN ceramic matrix, which may be owning to the fact that $\mathrm{Si}$ becomes more easily to bond with $\mathrm{C}$ rather than with $\mathrm{N}$ due to the insertion of MWCNTs [16]. The C1s peaks of sample 0 and sample 10 can be deconvoluted into $\mathrm{C}-\mathrm{C}$, Si-C and $\mathrm{C}-\mathrm{O}$ peaks (Fig. 6 0-3 and Fig. 6 10-3). In comparison with sample 0 , the amount of $\mathrm{Si}-\mathrm{C}$ peaks in sample 10 increased significantly (Fig. 6 0-3 and Fig. 6 10-3) [9], indicating that MWCNTs were embedded into SiCN ceramics by bonding with $\mathrm{Si}$, and that MWCNTs had good interface bonding with the ceramic matrix [9], which agrees with the SEM analysis results.
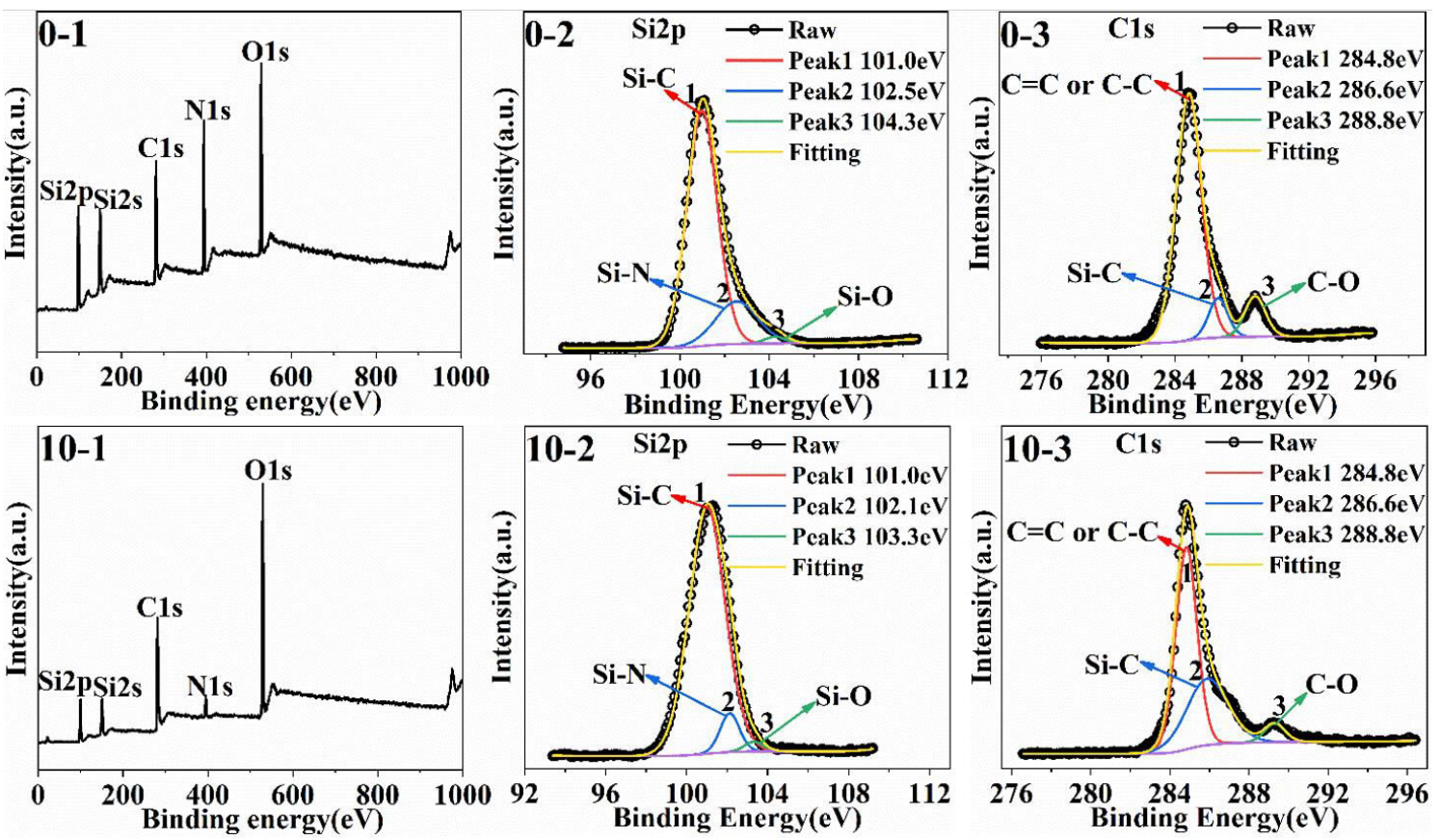

Fig. 6 XPS Spectra of Sample 0: Survey spectrum (0-1), narrow scan spectra of Si2p (0-2) and C1s (0-3); XPS Spectra of Sample 10: Survey spectrum (10-1), narrow scan spectra of Si2p (10-2) and C1s (10-3)

\subsection{EMW Absorption Performance Analysis}

According to the transmission line theory, the reflection loss (RL) of each sample 
can be calculated to characterize its EMW absorption performance. The specific value of RL can be calculated according to the Eq. (1) and Eq. (2) [20,21].

$$
\begin{aligned}
& Z=Z_{\text {in }} / Z_{0}=\sqrt{\frac{\mu_{r}}{\varepsilon_{r}}} \tanh \left[\mathrm{j}\left(\frac{2 \pi}{c}\right) \sqrt{\mu_{r} \varepsilon_{r}} \mathrm{fd}\right] \\
& \mathrm{RL}(\mathrm{dB})=20 \log \left|\left(Z_{\text {in }}-Z_{0}\right) /\left(Z_{\text {in }}+Z_{0}\right)\right|
\end{aligned}
$$

Where, $\mathrm{Z}$ is the impedance matching parameter, $Z_{\text {in }}$ is the wave impedance of the sample, $Z_{0}$ is the wave impedance of the free space, $\varepsilon_{r}$ is the complex permittivity of the sample, $\mu_{r}$ is the complex permeability, $\mathrm{d}$ is the thickness, $\mathrm{f}$ is the frequency, and $\mathrm{c}$ is the speed of light. If the calculated RL is less than $-10 \mathrm{~dB}$, it means that more than $90 \%$ of the EMW is absorbed. The data with $\mathrm{RL}<-10 \mathrm{~dB}$ is referred to as effective absorption data, and the corresponding bandwidth for $\mathrm{RL}<-10 \mathrm{~dB}$ is called EAB.

The calculated RL of each sample at different thickness and the 3D projection and 3D images of RL are shown in Fig. S1. By comparing and observing Fig. S1, it was observed that in sample 0, there was no EAB in the 3D projection (Fig. S1 0-2), and the minimum $\mathrm{RL}\left(\mathrm{RL}_{\mathrm{min}}\right)$ at $11 \mathrm{GHz}$ was about $-3.5 \mathrm{~dB}$ (Fig. S1 0-1) when the thickness was fixed at $4 \mathrm{~mm}$, indicating that about $55.3 \%$ of EMW is absorbed at this frequency band. The eddy carbon contained in the $\mathrm{SiCN}$ ceramic matrix is the basis for it to have EMW absorption performance to some extent [3, 4, 18]. After 3wt\% MWCNTs were added to the ceramic matrix, sample 3 had no EAB (Fig. S1 3-2), but its $\mathrm{RL}_{\mathrm{min}}$ decreased to $-5.8 \mathrm{~dB}$ at $9.2 \mathrm{GHz}$ at a thickness of $4 \mathrm{~mm}$ (Fig. S1 3-1), indicating that about $73.7 \%$ of EMW is absorbed at this frequency band. The addition of MWCNTs introduced new independent conductor units into ceramics, which 
increased the conduction loss and improved the EMW absorption performance of the material [9]. By increasing the addition amount of MWCNTs, EAB started to appear in the 3D projection of sample 5 (Fig. S1 5-2), and the EAB was $1.0 \mathrm{GHz}$ at a thickness of $5 \mathrm{~mm}$ (Fig. S1 5-2), indicating that increase in the number of independent conductor units leads to the enhance of conduction loss and the emergence of EAB. By further increasing the addition amount of MWCNTs, the EAB of sample 7 was expanded to $2.2 \mathrm{GHz}$ at a thickness of $5 \mathrm{~mm}$ (Fig. S1 7-2), indicating that the added MWCNTs still play the role of increasing the number of independent conductor units in the material and increasing the conduction loss. By continuing to rise the addition amount of MWCNTs, the EAB of sample 9 increased to $2.3 \mathrm{GHz}$ at a thickness of 5 $\mathrm{mm}$, and discontinuous effective absorption regions were found at near the sample thickness equal to $\lambda / 4$ (Fig. S1 9-2). When the addition amount of MWCNTs was further increased, the EAB of sample 10 showed a sudden jump, which rushed to 4.7 $\mathrm{GHz}$ at a thickness of $1.7 \mathrm{~mm}$. Compared with sample 9, the thickness of sample 10 decreased by $3.3 \mathrm{~mm}$, with the EAB increased by 104\% (Fig. S1 10-2). The sudden change of $\mathrm{EAB}$ indicates the arrival of conductive percolation state inside the ceramic: After the number of MWCNT independent conductor units in the matrix reaches a certain value, they begin to lap each other to form conductive percolation network, which greatly reduces the resistivity of the composite material, thus facilitates the rapid and continuous transfer of electrons in conductive pathways and the generation of joule heat, resulting in a sudden increase in the conductivity and dielectric property of composites, and finally leads to a sudden jump in the EMW absorption 
performance $(\mathrm{EAB})[22,23]$. On the basis of the percolation state, we continued to increase the addition amount of MWCNTs, and the EAB of sample 15 was observed to decrease instead of increasing: its $\mathrm{EAB}$ was $3.7 \mathrm{GHz}$ at a thickness of $1.3 \mathrm{~mm}$, and 2.1 GHz at a thickness of $2.8 \mathrm{~mm}$ (Fig. S1 15-2). The change trend of EAB was reversed, possibly because as the number of independent conductor units continues to grow, they will further intertwine and agglomerate in the conductive network; although this allows the conductivity of composite ceramics to keep growing, but too much conductivity can lead to impedance mismatch problem: incident waves are reflected at the air-material interface with impedance mismatch, which greatly reduces the likelihood of their entering the materials and being consumed, and the EAB is narrowed accordingly [2, 14]. The addition amount of MWCNTs continued to increase to $20 \mathrm{wt} \%, 25 \mathrm{wt} \%$ and even $30 \mathrm{wt} \%$, and similar to sample 15 , the EAB of samples 20, 25 and 30 tended to be narrowed gradually: the EAB of sample 20 was $1.8 \mathrm{GHz}$ at a thickness of $2.2 \mathrm{~mm}$ (Fig. $\mathbf{S 1 ~ 2 0 - 2}$ ); the EAB of sample 25 was $1.7 \mathrm{GHz}$ at a thickness of $2.6 \mathrm{~mm}$ (Fig. S1 25-2); the EAB of sample 30 was $1.5 \mathrm{GHz}$ at a thickness of $2.9 \mathrm{~mm}$ (Fig. S1 30-2). The EAB of samples 15, 20, 25 and 30 at different thickness were all smaller than that of sample 10. According to the above analysis of $\mathrm{EAB}$, it was speculated that a conductive percolation state was achieved inside the ceramic when the addition amount of MWCNTs was 10wt\% (by this time, composite ceramics exhibited the best EMW absorption performance), and further increasing its content would only lead to impedance mismatch and decrease of EAB.

\subsection{EMW Absorption Mechanism Analysis}


The impedance matching parameter $\mathrm{Z}$ represents the matching degree between material impedance and free space impedance. The closer its value is to 1 , the better the impedance matching between the two is, and the greater the probability of EMW entering the material to be consumed [9]. In order to explore the relationship among the addition amount of MWCNTs, impedance matching performance of composite ceramics and EAB, we calculated the $\mathrm{Z}$ (Fig. S2 Z•0 Z Z•30) of each sample at different frequencies and thickness by the Eq. (1), and attached the 3D projection of RL (Fig. S2 RL·0 RL·30) behind it, to analyze the effect of $\mathrm{Z}$ on EAB. For $0.8 \leq \mathrm{Z} \leq 1.3$, the data within this interval were temporarily referred to as the effective impedance matching data, and their corresponding regions in the $2 \mathrm{D}$ platform representation of $\mathrm{Z}$ were called the effective impedance matching band (EZB) $[4,9]$. In Fig. S2 two obvious EZBs were observed in the $\mathrm{Z}$ images of samples 0 9 (as shown in Fig. S2 Z•0 Z•9), but no EAB was found at the frequency and thickness where the two bands were located (Fig. S2 RL·0 RL・9), which shows that it is likely the insufficient electromagnetic attenuation capacity rather than impedance mismatch that leads to poor overall EMW absorption performance of composite ceramics when the addition amount of MWCNTs is between 0wt\% and 9wt $\%$ : the incident EMW can enter the material to a large extent, but pierces through the material as transmitted waves because it cannot be effectively attenuated inside the material, resulting in the deteriorated material performance. When the addition amount of MWCNTs was $10 \mathrm{wt} \%$, two EZBs changed to one, which was located near the sample thickness equal to $\lambda / 4$ (Fig. S2 Z $\mathbf{~ 1 0}$ ). At this time, the EAB of sample 10 also appeared near the 
thickness equal to $\lambda / 4($ Fig. $\mathbf{S 2} \mathbf{R L} \cdot \mathbf{1 0})$, and its range was covered in the range of EZB, indicating that when the addition amount of MWCNTs was $10 \mathrm{wt} \%$, appropriate impedance matching was one of the reasons for excellent EMW absorption performance of composite ceramics. When the addition amount of MWCNTs exceeded $10 \mathrm{wt} \%$, we found that the location of EZB did not move significantly with the increase of MWCNTs content, but the area of EZB decreased gradually (Fig. S2 Z•15 Fig. S2 Z•30), and the EAB of the samples also decreased gradually (Fig. S2 RL·15 Fig. S2 RL·30), which suggests that there is a good synergistic relationship between impedance matching and $\mathrm{EAB}$ of composite ceramics with the addition amount of MWCNTs at 10wt $\%$, and impedance matching gets worse by further rising the content of MWCNTs, thus further deteriorating the EMW absorption performance of ceramics. This conclusion accords with the speculation on the cause of EAB narrowing in section 3.4.

Complex permittivity $\left(\varepsilon_{r}=\varepsilon^{\prime}-\mathrm{j} \varepsilon^{\prime \prime}\right)$ and complex permeability $\left(\left(\mu_{r}=\mu^{\prime}-\mathrm{j} \mu^{\prime \prime}\right)\right.$ are basic parameters to analyze the EMW absorption performance. Where $\varepsilon^{\prime}$ and $\mu^{\prime}$ are the real parts of permittivity and permeability, which represent the storage capacity of the sample to electric and magnetic energy, respectively. $\varepsilon^{\prime \prime}$ and $\mu$ " are the imaginary parts of permittivity and permeability, which represent the theoretical loss capacity of the sample to electric and magnetic energy, respectively. $\varepsilon^{\prime \prime} / \varepsilon^{\prime}$ and $\mu^{\prime \prime} / \mu^{\prime}$ are the dielectric loss tangent $\left(\tan \delta_{\varepsilon}\right)$ and magnetic loss tangent $\left(\tan \delta_{\mu}\right)$, which represent the actual dissipation capacity of the sample to electric and magnetic energy, respectively. Since neither SiCN ceramics nor MWCNTs contain magnetism, it is speculated that 
dielectric loss makes a key contribution to the attenuation of EMW energy. The establishment of dielectric theory has aroused the attention of many researchers to the dielectric properties of the mixture composed of multiple dielectrics. Due to the interaction between different phases and the accumulation of space charges at the interface between different phases, which further leads to the formation of local fields, the mixture shows an effective permittivity $\left(\varepsilon_{m}\right)$ at the macro level, which can be reckoned by the logarithmic mixing rule (Eq. (3)) given by Lichtenecker [24-26]:

$\left(\varepsilon_{m}\right)^{n}=\sum_{i} v_{i}\left(\varepsilon_{i}\right)^{n}$

Where, $v_{i}$ is the volume fraction of each single phase, $\varepsilon_{i}$ is the permittivity of each single phase, and $\mathrm{n}$ is a constant between -1 and 1 . Due to the difference in the angle between the arrangement direction of dielectrics and the direction of applied electric field, $\varepsilon_{m}$ of composite materials composed of multiple dielectrics can be calculated by the following three ways: 1 . When the dielectric layers are in the same direction as the applied electric field (Fig. 7a), the mixture as a whole can be considered as a parallel circuit. According to the electric field intensity of each layer and the Eq. (4), $\varepsilon_{m}$ of the composite material can be obtained:

$\varepsilon_{m}=\sum_{i} v_{i} \varepsilon_{i}$

2. When each dielectric layer is perpendicular to the direction of the applied electric field (Fig. 7b), the mixture as a whole can be regarded as a series circuit. According to the electric field intensity of each layer and the Eq. (5), $\varepsilon_{m}$ of the composite material can be obtained:

$\frac{1}{\varepsilon_{m}}=\sum_{i} \frac{v_{i}}{\varepsilon_{i}}$ 
Eq. (4) and Eq. (5) are two extreme cases of the Eq. (3), with n values of 1 and -1, respectively.

3. Considering different geometric shapes, concentrations and distributions of dielectrics (Fig. 7c), different values of $n$ were selected; Maxwell derived the Eq. (6) to calculate $\varepsilon_{m}$ of composite materials with irregular states of inclusions [25,26]:

$\varepsilon_{m}=\frac{v_{1} \varepsilon_{1}\left(\frac{2}{3}+\frac{\varepsilon_{2}}{3 \varepsilon_{1}}\right)+v_{2} \varepsilon_{2}}{v_{1}\left(\frac{2}{3}+\frac{\varepsilon_{2}}{3 \varepsilon_{1}}\right)+v_{2}}$

When the inclusions are materials with low dielectric loss that have adjacent permittivity with the matrix, the result of the Eq. (6) is close to that of the logarithmic mixing rule, indicating that in this case $\varepsilon_{m}$ is the average of components in the composite material [25]. When the inclusions are materials with high dielectric loss that have higher permittivity than the matrix (such as conductive CNTs), once the concentration of inclusions reaches the percolation threshold, dielectric percolation will occur inside the material, leading to a very sudden change in dielectric behavior. In this case, due to the dielectric percolation and the interaction between high dielectric loss materials, the permittivity of the composite material can be higher than that of any component [10-12]. The inclusions in this study were MWCNTs (highly conductive materials). For the purpose of exploring the influence of its concentration on dielectric properties of composite ceramics, electromagnetic parameters of each sample were tested, and the results are shown in Fig. 8. 


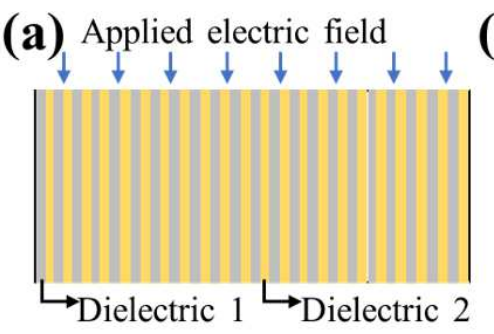

(b) Applied electric field

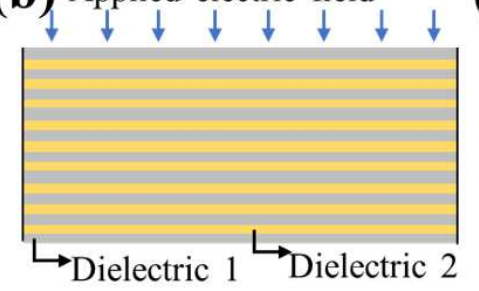

(c) Applied electric field

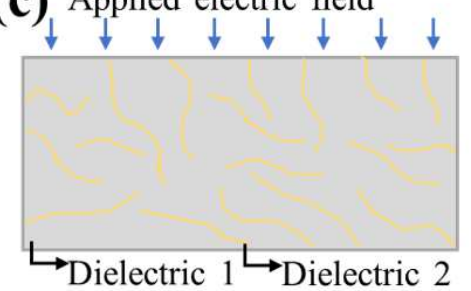

Fig. 7 Distribution between Filler Dielectric and Dielectric Matrix: Parallel to each other and parallel to the direction of the electric field (a), parallel to each other and perpendicular to the direction of the electric field (b), fillers are randomly distributed in the matrix, and the two have no definite angle relation with the direction of the electric filed (c)

According to Fig. 8a, when the addition amount of MWCNTs was $\leq 9 \mathrm{wt} \%, \varepsilon^{\prime}$ hardly fluctuated with the change of frequency, and the image presented as a straight line. With rising the addition amount of MWCNTs, the value of $\varepsilon^{\prime}$ gradually rose slightly. When the addition amount of MWCNTs was increased to $10 \mathrm{wt} \%$, the $\varepsilon^{\prime}$ of the samples increased significantly (the addition amount of MWCNTs increased by $1 \mathrm{wt} \%$, and the $\varepsilon^{\prime}$ increased by 1.5 times $)$, frequency dispersion $\left(2 \sim 17 \mathrm{GHz}\right.$, the value of $\varepsilon^{\prime}$ decreased gradually with the increase of frequency) and chromatic dispersion (17 18 $\mathrm{GHz}$, the value of $\varepsilon^{\prime}$ went up with the increase of frequency) were observed [2]. Frequency dispersion is conducive to the coordination of impedance matching of composite ceramics, and chromatic dispersion is accompanied by the loss of energy as is the process of resonance $[2,21]$. The sudden change of the $\varepsilon^{\prime}$ of sample 10 may be related to the formation of conductive network and percolation state in the ceramics [14]. When the addition amount of MWCNTs was greater than $10 \mathrm{wt} \%, \varepsilon^{\prime}$ continued to rise with the increase of addition amount, indicating that the storage capacity of the 
samples to electric energy continued to increase, but it is worth noting that the advantage of impedance matching is usually limited by a higher $\varepsilon^{\prime}$ [27]. Fig. 8b shows the change of $\varepsilon^{\prime \prime}$ of each sample. According to the figure, when the addition amount of MWCNTs was $\leq 9 \mathrm{wt} \%$, the $\varepsilon^{\prime \prime}$ of the sample hardly fluctuated with the change of frequency and almost showed a straight line. With the increase of MWCNTs addition amount, $\varepsilon^{\prime \prime}$ gradually increased slightly (as the addition amount of MWCNTs rose from $3 w t \%$ to $5 w t \%$, $\varepsilon^{\prime \prime}$ increased by 0.68 ; if the addition amount of MWCNTs rose from $5 \mathrm{wt} \%$ to $9 \mathrm{wt} \%, \varepsilon "$ increased by 0.29$)$, which indicates that the increase in the content of MWCNTs at this time plays a role in enhancing the dielectric loss of composite ceramics, but the effect is unconspicuous. When the addition amount of MWCNTs reached $10 \mathrm{wt} \%$, the $\varepsilon^{\prime \prime}$ of the sample rose sharply (if the addition amount of MWCNTs lifted by $1 w t \%, \varepsilon "$ rose by 2.46 ), and with the increase of frequency in the range of $16 \sim 18 \mathrm{GHz}, \varepsilon$ " showed a downward trend, indicating that an obvious conduction loss occurred in the sample at this time [28]. The sudden increase of $\varepsilon^{\prime \prime}$ and the clear presentation of conduction loss are associated with that a state of conductive percolation is achieved inside the material at this time $[14,22,23]$. When the addition amount of MWCNTs was $>10 \mathrm{wt} \%$, $\varepsilon^{\prime \prime}$ was on the rise overall with the increase of addition amount. In the range of $6 \sim 10 \mathrm{GHz}$, the slope of the $\varepsilon^{\prime \prime}$ curve gradually increased, indicating that the carrier concentration in the material increased [2,28]. Compared with samples $0 \sim 10$, a special phenomenon appeared in $\varepsilon^{\prime \prime}$ of samples 15 30: in the range of $10 \sim 12 \mathrm{GHz}$, it rose with the increase of frequency, which is presumably caused by that the contribution to dielectric loss of direct current 
loss is greater than that of alternating current loss [29]. The behavior of $\varepsilon^{\prime \prime}$ also changed abruptly when the addition amount of MWCNTs was $10 \mathrm{wt} \%$, indicating that the content of MWCNTs in the material reached the percolation threshold at this time. Fig. 8c shows the $\tan \delta_{\varepsilon}$ of each sample, which characterizes the dissipation capacity of the material to electric energy in EMW. By comparing Fig. 8b and Fig. 8c, $\tan \delta_{\varepsilon}$ had a similar change rule with $\varepsilon^{\prime \prime}, \tan \delta_{\varepsilon}$ of samples 3 30 was higher than that of sample 0 , indicating that the addition of MWCNTs plays a positive role in improving the dielectric loss performance of composite ceramics. When the addition amount of MWCNTs was $\leq 9 \mathrm{wt} \%, \tan \delta_{\varepsilon}$ fluctuated slightly with the change of frequency, and $\tan \delta_{\varepsilon}$ gradually increased slightly with rising addition amount of MWCNTs. When the addition amount of MWCNTs increased to $10 \mathrm{wt} \%, \tan \delta_{\varepsilon}$ rose dramatically, with the maximum increased to 0.41 . By continuing to increase the addition amount of MWCNTs, $\tan \delta_{\varepsilon}$ began to decrease instead of increasing in the range of some frequency bands (8 11 GHz, Fig. 8c). According to comprehensive analysis of Fig. 8 $\mathbf{a}, \mathbf{b}, \mathbf{c}$, we found that when the addition amount of MWCNTs was $10 \mathrm{wt} \%$, an abrupt change occurred at $\varepsilon^{\prime}, \varepsilon^{\prime \prime}$ and $\tan \delta_{\varepsilon}$ of the samples, demonstrating that MWCNTs lapped and interacted with each other in the ceramics at this time, resulting in a conductive percolation state inside the ceramics, which further triggered the dielectric percolation and the abrupt change in and dielectric behavior [10-12,22,23]. This conclusion also accounts for the sudden increase in EAB of sample 10. From the above analysis, when the addition amount of MWCNTs was 10wt\%, the EMW absorption performance parameter (EAB) and dielectric loss behavior of composite 
ceramics changed dramatically. Therefore, the percolation threshold of MWCNTs in composite ceramics was believed to be $10 \mathrm{wt} \%$. Since neither the ceramic matrix nor MWCNTs contain magnetism, $\mu "$ and $\tan \delta_{\mu}$ were close to 0 (Fig. 8e and Fig. 8f) as the $\mu^{\prime}$ of all samples was close to 1 (Fig. 8d). By comparing Fig. 8c and Fig. 8f, dielectric loss made a substantial contribution to EMW attenuation, while the contribution of magnetic loss was negligible. Hence, complex permeability parameters of samples are not elaborated here.
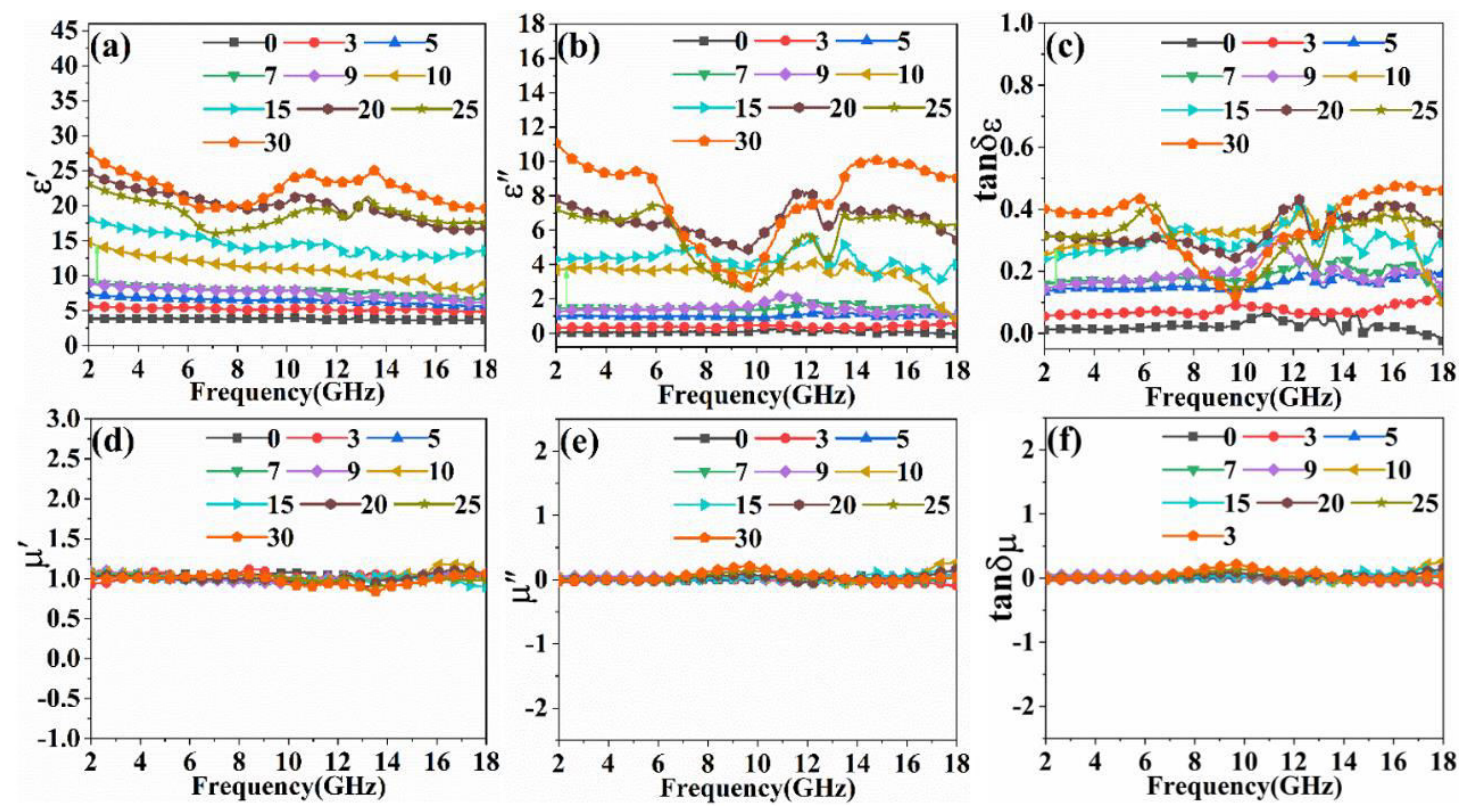

Fig. $8 \varepsilon^{\prime}(\mathrm{a}), \varepsilon^{\prime \prime}(\mathrm{b}), \tan \delta_{\varepsilon}(\mathrm{c}), \mu^{\prime}(\mathrm{d}), \mu^{\prime \prime}(\mathrm{e})$ and $\tan \delta_{\mu}(\mathrm{e})$ of $\operatorname{SiCN}(\mathrm{MWCNTs})$

In order to further explore the dielectric loss behavior of the samples, we introduced the Debye relaxation model to analyze the dielectric loss mechanism in the material. If the polarization of the material follows the Debye relaxation during the interaction with EMW, then its $\varepsilon^{\prime \prime}-\varepsilon^{\prime}$ curve should be a semicircle (Cole semicircle), and each semicircle represents a polarization process that follows the Debye relaxation [4,21]. Fig. 9 shows the $\varepsilon^{\prime \prime}-\varepsilon^{\prime}$ images of sample 0 and sample 10. By comparing Fig. 9a and Fig. 9b, obvious Cole semicircles appeared in sample 10 
compared with sample 0 , indicating that the addition of MWCNTs provided abundant interfacial polarization for composite ceramics [30,31]. Cole semicircles were mainly distributed at higher frequencies, indicating that interfacial polarization is the main mechanism of dielectric loss at higher frequencies [4]. In the lower frequencies, the $\varepsilon^{\prime \prime}-\varepsilon^{\prime}$ image of sample 10 presented nearly as a straight line, indicating that conduction loss is the main way to dissipate electric energy at lower frequencies $[22,23,32]$. The occurrence of conduction loss is related to the formation of the conductive percolation state in the sample at this time [22, 23, 32]. In addition, a certain deviation existed between the Cole semicircle of sample 10 and the standard semicircle, indicating that there are other polarization or relaxation behaviors besides interfacial polarization, such as dipole relaxation [9]. There were abundant intrinsic dipoles in the ceramic matrix and MWCNTs, which will spin as the direction of the alternating electric filed changes, leading to dipole relaxation and further energy loss [9]. The above analysis of $\varepsilon^{\prime \prime}-\varepsilon^{\prime}$ image showed that interfacial polarization, conduction loss and dipole relaxation are main mechanisms for the dielectric loss of composite ceramics 10 .
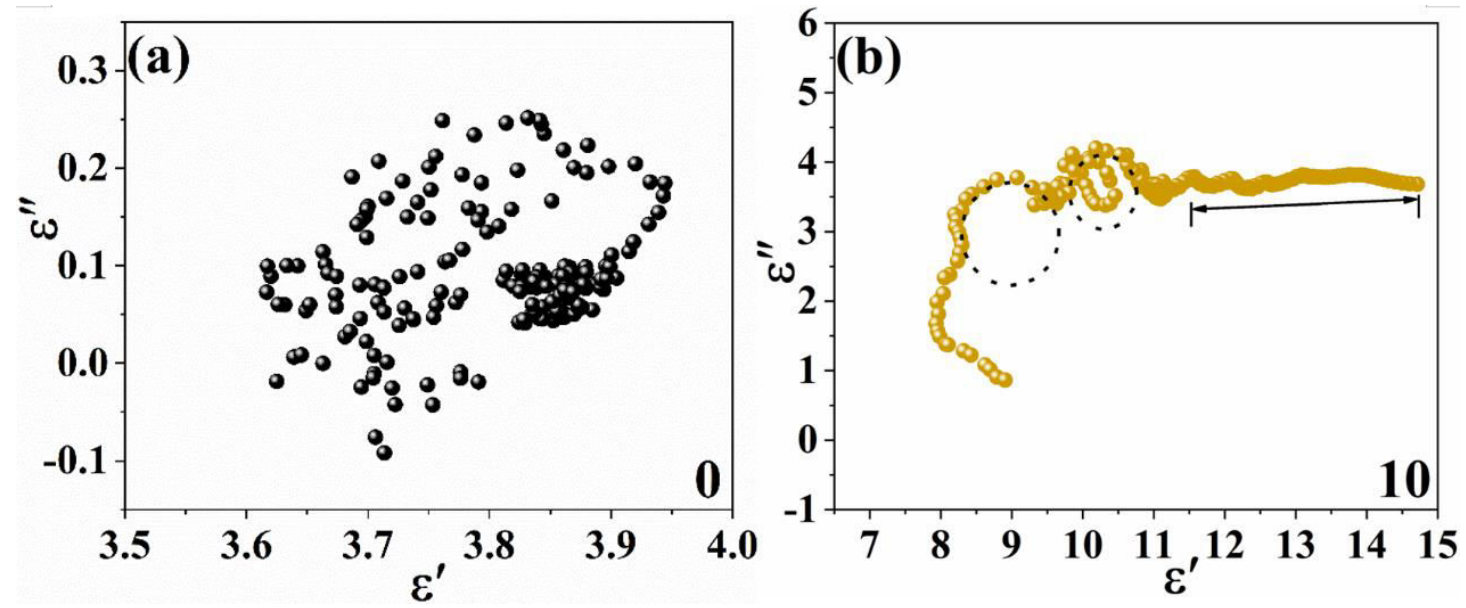

Fig. 9 Cole-Cole Semicircle Image of Sample 0 (a) and Sample 10 (b) 
Besides impedance matching and dielectric loss performance, the thickness $\left(t_{m}\right.$, which can be calculated by the Eq. (5)) and the attenuation constant ( $\alpha$, which can be calculated by the Eq. (6)) of the material are also important parameters that affect its EMW absorption performance [33]. The effect of thickness on the EMW absorption performance of the material can be explained by $\frac{n \lambda}{4}(n=1,3,5 \ldots)$ matching model (Eq. (7)):

$t_{m}=\frac{n \lambda}{4}=\frac{n c}{4 f_{m \sqrt{\left|\mu_{r} \| \varepsilon_{r}\right|}}}(\mathrm{n}=1,3,5,7 \ldots)$

Where, $t_{m}$ is the thickness of the sample, $\lambda$ means the wavelength of EMW, $f_{m}$ represents the corresponding frequency at the peak on the RL curve, and other parameters have the same meaning as above. If the sample thickness conforms to the n $\lambda / 4$ matching model in the Eq. (7) (i.e., the sample thickness is $n / 4$ times the wavelength of EMW), the incident EMW penetrating through the air-material interface interferes destructively with the reflected EMW from the material bottom, which maximizes the attenuation of EMW [4]. Fig. 10a is the RL of sample 10 at different thickness, from which the corresponding (frequency, thickness) points (i.e. $\left(f_{m}, t_{m}\right)$ point, drawn with colored points in Fig. 10b) at the peak of curve in Fig. 10a were observed to be in good agreement with the $\lambda / 4$ curve (Fig. 10b), which shows that the $\lambda / 4$ thickness matching condition also makes an indispensable contribution to the attenuation of EMW for the sample. In addition, we also found that the $\mathrm{Z}$ value corresponding to the peak of the RL curve was close to 1 (Fig. 10c), which shows that the $\lambda / 4$ thickness matching condition and appropriate impedance matching are well coordinated and work together to contribute to the excellent EMW 
absorption performance of composite ceramics. $\alpha$ (calculated by the Eq. (8)) refers to the overall attenuation capacity of the material to EMW energy, and a larger value suggests a greater EMW attenuation of the material.

$\alpha=\left(\frac{\pi \mathrm{f}}{\mathrm{c}}\right) \sqrt{2 \mu^{\prime} \varepsilon^{\prime}} \sqrt{\tan \delta_{\varepsilon} \tan \delta_{\mu}-1+\sqrt{\left(\tan \delta_{\varepsilon}{ }^{2}+1\right)\left(\tan \delta_{\mu}{ }^{2}+1\right)}}$

Fig. 10e shows $\alpha$ of each sample. It can be observed from the figure that as the addition amount of MWCNTs increased, $\alpha$ was on the rise on the whole, indicating that the increase of MWCNTs content will improve the EMW attenuation capacity of materials. Similar to the change rule of permittivity, when the addition amount of MWCNTs increased from $9 \mathrm{wt} \%$ to $10 \mathrm{wt} \%$ (percolation threshold), $\alpha$ of sample 10 increased suddenly, up to $224.81,1.8$ times that of sample 9, showing its excellent EMW attenuation performance. This result tallies with the previous analysis of EAB and permittivity.

Table 2 Comparison of performance parameters of composite ceramics after introducing different fillers into SiCN ceramics

\begin{tabular}{llllll}
\hline Fillings & $\begin{array}{l}\text { Filling } \\
\text { rate(wt\%) }\end{array}$ & $\begin{array}{l}\text { Sample } \\
\text { thickness } \\
(\mathrm{mm})\end{array}$ & $\mathrm{RL}_{\min }(\mathrm{dB})$ & $\mathrm{EAB}(\mathrm{GHz})$ & Ref \\
\hline $\mathrm{Fe}_{2} \mathrm{O}_{3}$ & 15 & 2 & -11 & $<1$ & 34 \\
$\mathrm{Fe}_{2} \mathrm{O}_{3}$ and $\mathrm{Eu}_{2} \mathrm{O}_{3}$ & 60 & 2 & -12 & $<1(0.7)$ & 35 \\
$\mathrm{Co}$ & 10 & 2 & -8 & $<1$ & 36 \\
$\mathrm{Co}(\mathrm{acac})_{3}$ & 60 & 2 & -14 & $<1$ & 37 \\
$\mathrm{Ni}(\mathrm{II})$ complex & 30 & 4 & -11 & $<1$ & 15 \\
$\mathrm{Ni}$ & 20 & 2 & -23 & 2 & 5 \\
$\mathrm{Fe}(\text { acac })_{3}$ & 50 & 4 & -10.5 & 3.3 & 18 \\
$\mathrm{MWCNTs}$ & 10 & 1.7 & -21.7 & 4.7 & This work \\
\hline
\end{tabular}

Based on the above analysis, the EMW absorption performance (EAB), Z, $\varepsilon^{\prime}, \varepsilon^{\prime \prime}$, 
$\tan \delta_{\varepsilon}$ and $\alpha$ of SiCN (MWCNTs) composite ceramics changed abruptly when the addition amount of MWCNTs was $10 \mathrm{wt} \%$, which suggests that the percolation threshold of MWCNTs in composite ceramics was 10wt\%. Sample 10 had an EAB of 4.7 $\mathrm{GHz}$ at a thickness of $1.7 \mathrm{~mm}$ (Fig.S1 10-2), and the EAB covering $\mathrm{C}, \mathrm{X}$ and $\mathrm{Ku}$ bands can be obtained by adjusting its thickness from $1.0 \mathrm{~mm}$ to $5.0 \mathrm{~mm}$ (Fig. 10a). Compared with other similar SiCN-based composite ceramics, SiCN (MWCNTs) composite ceramics have significantly improved the EAB while reducing its thickness (Table 2) $[5,15,18,34-37]$. The excellent EMW absorption performance is the result of coordination and joint action of multiple mechanisms, rather than a single mechanism acting alone. The EMW absorption mechanism of sample 10 can be schematically expressed by Fig. 11, which can be specifically summarized as below: First, good impedance matching enables the maximum EMW to enter the material; Second, conductive network in the material promotes the rapid and continuous electron transport, during which part of electric energy is converted into heat energy and dissipated. At the heterogeneous interface between SiCN ceramics and MWCNTs is easily formed a local field under the action of electric field, by the action of which atoms at the interface deviate from the original position, that is, a polarization process occurs, resulting in interfacial polarization loss. Intrinsic dipoles in $\mathrm{SiCN}$ ceramics and MWCNTs spin as the direction of the alternating electric field changes, because the spin speed of some dipoles will lag behind the speed at which the direction of the alternating electric field changes, so the spin has a certain hysteresis, that is, spin relaxation occurs, resulting in relaxation loss. Finally, the EMW reflected from the 
bottom of the material interferes destructively with the incident EMW as much as possible due to the $\lambda / 4$ thickness matching condition, reducing the amount of reflected EMW. Owing to the coordination and combined action of a variety of EMW attenuation mechanisms, SiCN (MWCNTs) composite ceramics exhibit excellent EMW absorption performance.

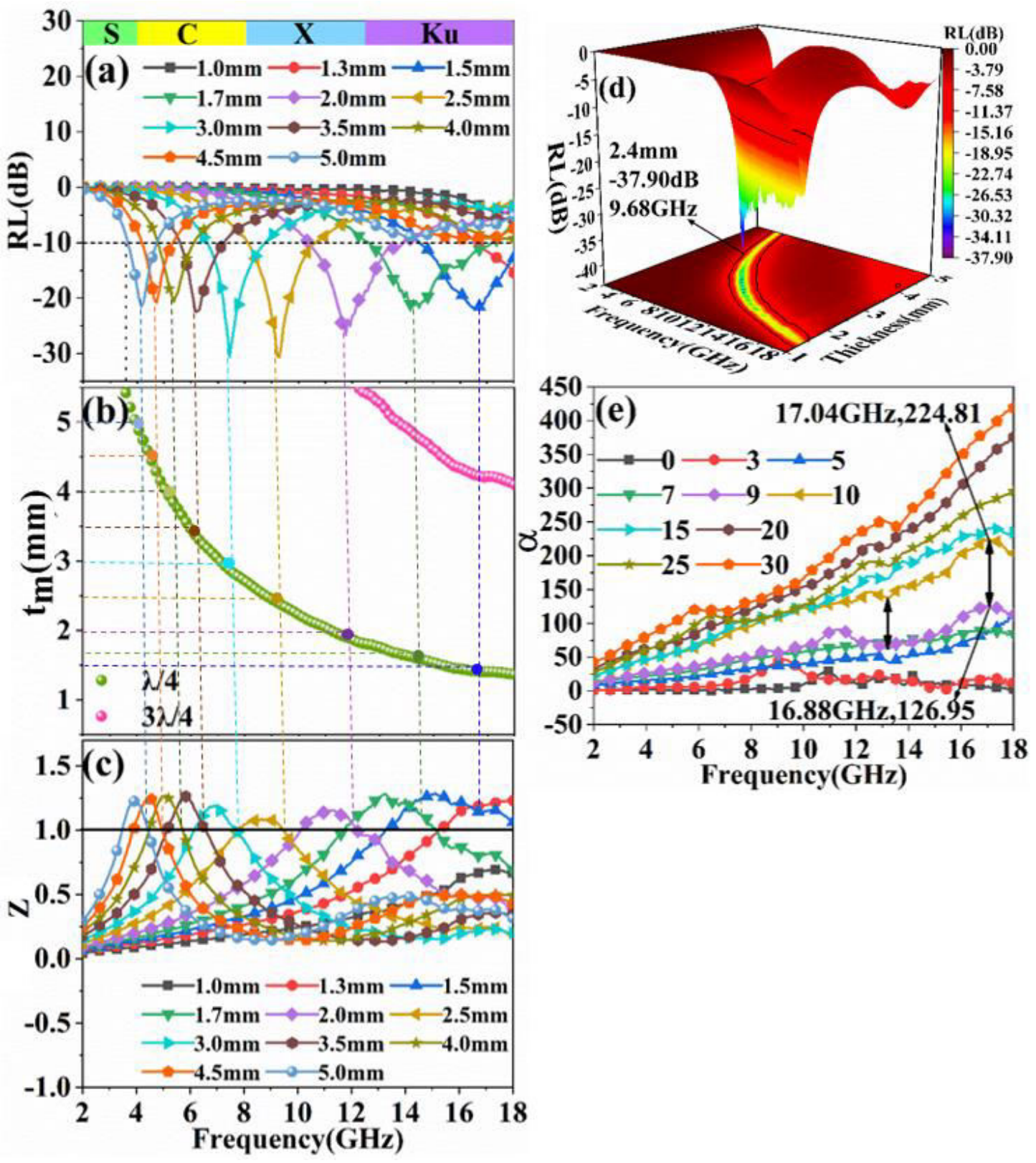

Fig. 10 RL of Sample 10 under Different Thickness (a) and Simulation of Thickness and Frequency under n $\lambda / 4$ Condition (b), Z (c), 3D Diagram of RL (d) and $\alpha$ (e) 

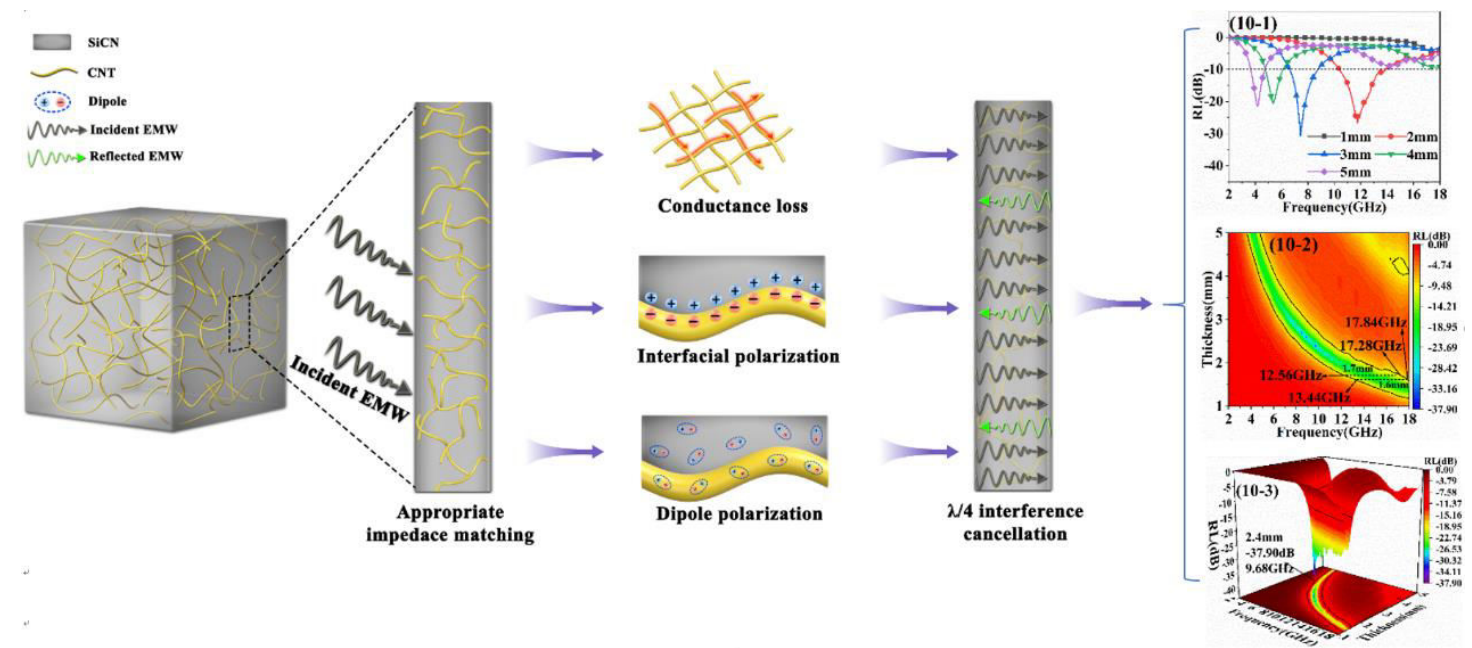

Fig. 11 EMW Absorption Mechanism Diagram of Sample 10

\section{Conclusion}

A polymer derivation method was employed in this paper to prepare a series of SiCN (MWCNTs) composite ceramics by adjusting the mass ratio of MWCNTs. The effect of the addition amount of MWCNTs on the RL, EAB, permittivity, $Z$ and $\alpha$ of composite ceramics was discussed, the percolation threshold of the corresponding MWCNTs when composite ceramics exhibit the optimal EWM absorption performance was studied, and the EMW absorption mechanism of composite ceramics (with the widest $\mathrm{EAB}$ ) when the MWCNTs addition amount was $10 \mathrm{wt} \%$ was analyzed. The results of analysis indicate that the EAB, Z, $\varepsilon^{\prime}, \varepsilon^{\prime \prime}, \tan \delta_{\varepsilon}$ and $\alpha$ of composite ceramics changed abruptly when the addition amount of MWCNTs was $10 \mathrm{wt} \%$, which suggests that the percolation threshold of MWCNTs in composite ceramics was $10 \mathrm{wt} \%$, and the composite ceramics (sample 10) obtained at this time had the best EMW absorption performance: its $\mathrm{RL}_{\min }$ was $-37.9 \mathrm{~dB}$, and the $\mathrm{EAB}$ reached $2.8 \mathrm{GHz}$ at a thickness of $2.4 \mathrm{~mm}$; its $\mathrm{RL}_{\min }$ was $-21.7 \mathrm{~dB}$, and the $\mathrm{EAB}$ reached $4.7 \mathrm{GHz}$ at a thickness of $1.7 \mathrm{~mm}$. By changing the thickness of sample 10 
from $1.0 \mathrm{~mm}$ to $5.0 \mathrm{~mm}$, the $\mathrm{EAB}$ containing the $\mathrm{C}, \mathrm{X}$ and $\mathrm{Ku}$ bands can be acquired. Excellent EMW absorption performance is the result of the combined action from suitable impedance matching, good conduction loss, a certain degree of interfacial polarization loss and dipole relaxation loss, and $\lambda / 4$ thickness matching condition. In a word, the as-prepared SiCN (MWCNTs) composite ceramics can be expected to be a potential candidate in the field of light, efficient and broadband EMW absorbers.

\section{Acknowledgment}

This work was financially supported by the Jilin Provincial Development and Reform Commission Project (Grant No. 2021C036-1).

\section{Electronic Supplementary Material}

Supplementary material is available in the online version of this article.

\section{References}

[1] Yuan K, Han D, Liang J, et al. Microwave induced in-situ formation of SiC nanowires on SiCNO ceramic aerogels with excellent electromagnetic wave absorption performance[J]. Journal of Advanced Ceramics, 2021, 10(5): 0-0.

[2] Lyu L, Wang F, Zhang $\mathrm{X}$, et al. CuNi alloy/carbon foam nanohybrids as high-performance electromagnetic wave absorbers[J]. Carbon, 2021, 172: 488-496.

[3] Guo X, Feng Y, Lin X, et al. The dielectric and microwave absorption properties of polymer-derived SiCN ceramics[J]. Journal of the European Ceramic Society, 2018, 38(4): 1327-1333.

[4] Wang S, Ashfaq M Z, Gong H, et al. Electromagnetic wave absorption properties of magnetic particle-doped SiCN (C) composite ceramics[J]. Journal of Materials Science: Materials in Electronics, 2021, 32(4): 4529-4543.

[5] Liu Y, Feng Y, Gong H, et al. Electromagnetic wave absorption properties of nickel-containing polymer-derived SiCN ceramics[J]. Ceramics International, 2018, 44(9): 10945-10950.

[6] Feng Y, Qiu T, Li X, et al. Microwave absorption properties of the carbonyl iron/EPDM radar absorbing materials[J]. Journal of Wuhan University of Technology-Mater. Sci. Ed., 2007, 22(2): 266-270.

[7] Yang L, Zhang Y, Du S. Study of Chemical Reactivity of Doped Carbon Nanotubes by Simple Hückel Molecular Orbital Calculations with Matlab Programming[J]. Computational physics, 2017, 34(6): 685-696. 
[8] Mallikarjuna H M, Ramesh C S, Koppad P G, et al. Effect of carbon nanotube and silicon carbide on microstructure and dry sliding wear behavior of copper hybrid nanocomposites[J]. Transactions of Nonferrous Metals Society of China, 2016, 26(12):3170-3182.

[9] Wang S, Gong H, Zhang $\mathrm{Y}$, et al. Microwave absorption properties of polymer-derived $\mathrm{SiCN}(\mathrm{CNTs})$ composite ceramics[J]. Ceramics International, 2021, 47(1):1294-1302.

[10] Sihvola A. Mixing Rules with Complex Dielectric Coefficients[J]. Subsurface Sensing Technologies and Applications, 2000, 1(4):393-415.

[11] Bergman D J. The dielectric constant of a composite material-A problem in classical physics[J]. Physics Reports, 1978, 43(9):377-407.

[12] Sihvola A, Saastamoinen S, Heiska K. Mixing rules and percolation[J]. Remote Sensing Reviews, 1994, 9(1-2): 39-50.

[13] Gong C, Meng H, Zhao X, et al. Unique Static Magnetic and Dynamic Electromagnetic Behaviors in Titanium Nitride/Carbon Composites Driven by Defect Engineering[J]. Scientific Reports, 2016, 6(1):18927.

[14] Nan C W, Shen Y, Ma J. Physical properties of composites near percolation[J]. Annual Review of Materials Research, 2010, 40: 131-151.

[15] Liu Y, Feng $\mathrm{Y}$, Gong $\mathrm{H}$, et al. Microwave absorbing performance of polymer-derived $\mathrm{SiCN}(\mathrm{Ni})$ ceramics prepared from different nickel sources[J]. Journal of Alloys and Compounds, 2018, 749: 620-627.

[16] Feng Y, Feng N, Wei Y, et al. Preparation and improved electrochemical performance of $\mathrm{SiCN}$-graphene composite derived from poly(silylcarbondiimide) as Li-ion battery anode[J]. Journal of Materials Chemistry A, 2014, 2(12):4168-4177.

[17] Saleem A, Zhang Y, Gong H, et al. Enhanced thermal conductivity and mechanical properties of a GNP reinforced $\mathrm{Si}_{3} \mathrm{~N}_{4}$ composite[J]. RSC Advances, 2019, 9: 39986-39992.

[18] Feng Y, Guo X, Gong $\mathrm{H}$, et al. Microwave absorption performance of PDCs-SiCN $(\mathrm{Fe})$ ceramics with negative imaginary permeability[J]. Ceramics International, 2018,44(9): 10420-10425.

[19] Liu M, Cowley J M. Structure of carbon nanotubes studied by HRTEM and nanodiffraction[J]. Ultramicroscopy, 1994, 53(4):333-342.

[20] Liang H, Liu J, Zhang Y, et al. Ultra-thin broccoli-like SCFs@ $\mathrm{TiO}_{2}$ one-dimensional electromagnetic wave absorbing material[J]. Composites,2019,178(Dec.1):107507.1-107507.12.

[21] Lyu L, Wang F, Li B, et al. Constructing $1 \mathrm{~T} / 2 \mathrm{H} \mathrm{MoS}$ nanosheets/3D carbon foam for high-performance electromagnetic wave absorption[J]. Journal of Colloid and Interface Science, 2020, 586: 613-620.

[22] Wu M, Ni W, Hu J, et al. NASICON-Structured $\mathrm{NaTi}_{2}\left(\mathrm{PO}_{4}\right)_{3}$ for Sustainable Energy Storage[J]. Nano-Micro Letters, 2019, 11(1):44.

[23] Han J, Lu K., Yue Y, et al. Synthesis and electrochemical performance of flexible cellulose nanofiber-carbon nanotube/natural rubber composite elastomers as supercapacitor electrodes[J]. New Carbon Materials, 2018, 33(4): 341-350.

[24] Wakino K, Okada T, Yoshida N, et al. A New Equation for Predicting the 
Dielectric Constant of a Mixture[J]. Journal of the American Ceramic Society, 1993, 76(10): 2588-2594.

[25] Kingery, W. David, Harvey Kent Bowen, and Donald R. Uhlmann. Introduction to ceramics. Vol. 17. John wiley \& sons, 1976.

[26] Wang G Q, Shun-Hua W U, Zhao Y S, et al. 3-Dimensional Simulation of the Interior Electric Field and Macro Dielectric Constant of a Two-Phased Composite Material[J]. Journal of Inorganic Materials, 2004, 19(1):214-222.

[27] Du Y, Liu W, Qiang R, et al. Shell Thickness-Dependent Microwave Absorption of Core-Shell $\mathrm{Fe}_{3} \mathrm{O}_{4} @ \mathrm{C}$ Composites[J]. Acs Applied Materials \& Interfaces, 2014, 6(15):12997-13006.

[28] Chen H. Dielectric and ferroelectric properties of scandium tantalate crystals before and after gamma-ray irradiation. Journal of Shandong University, 1985,4:60-64.

[29] Yusoff A N, Abdullah M H, Ahmad S H, et al. Electromagnetic and absorption properties of some microwave absorbers[J]. Journal of Applied Physics, 2002, 92(2):876-882.

[30] Wu F, Xia Y, Wang Y, et al. Two-step reduction of self-assembed three-dimensional (3D) reduced graphene oxide (RGO)/zinc oxide ( $\mathrm{ZnO})$ nanocomposites for electromagnetic absorption[J]. Journal of Materials Chemistry A, 2014, 2(47):20307-20315.

[31] Yan C, Wei M, Li Z, et al. Towards outstanding dielectric consumption derived from designing one-dimensional mesoporous $\mathrm{MoO}_{2} / \mathrm{C}$ hybrid heteronanowires[J]. Journal of Materials Chemistry C, 2017, 5(35):8981-8987.

[32] Wei Y, Shi Y, Jiang Z, et al. High performance and lightweight electromagnetic wave absorbers based on TiN/RGO flakes[J]. Journal of Alloys and Compounds, 2019, 810:151950.

[33] Liu J, Liang H, Zhang Y, et al. Facile synthesis of ellipsoid-like $\mathrm{MgCo}_{2} \mathrm{O}_{4} / \mathrm{Co}_{3} \mathrm{O}_{4}$ composites for strong wideband microwave absorption application[J]. Composites Part B Engineering, 2019, 176:107240.

[34] Wang Y, Feng Y, Guo X, et al. Electromagnetic and wave absorbing properties of Fe-doped polymer-derived SiCN ceramics[J]. RSC Advances, 2017, 7(73):46215-46220.

[35] Yu L, Feng Y, Gong H, et al. Electromagnetic wave absorption properties of europium-doped $\mathrm{SiCN}(\mathrm{Fe})$ polymer-derived ceramics[J]. Journal of Materials Science Materials in Electronics, 2018, 29(14):12496-12502.

[36] Wang S, Mao J, Liu Y, et al. Electromagnetic Wave Absorption Properties of Cobalt-Containing Polymer-Derived SiCN Ceramics[J]. IOP Conference Series: Materials Science and Engineering, 2019, 678(1):012047 (12pp).

[37] Wang S, Gong H Y, Zhang Y, et al. Electromagnetic wave absorption properties of polymer derived $\mathrm{SiCN}$ ceramics doped with $\mathrm{Co}_{3} \mathrm{C}$ particles[J]. Optoelectronics and Advanced Materials-Rapid Communications, 2020, 14(November-December 2020): 528-537. 


\section{Supplementary Files}

This is a list of supplementary files associated with this preprint. Click to download.

- MEletronicSupplementMateria.1.docx 